\title{
Impact de l'allégeance en ses dimensions idéologique et mercenaire sur les réactions aux injustices professionnelles
}

\author{
Coralie Soudan ${ }^{1}$, Bernard Gangloff ${ }^{2}$
}

\section{Abstract}

Several studies have shown the existence of a norm of allegiance that we can define as the social valorization of the individuals avoiding to question the social environment, and in particular to question the powers hierarchy in a social system. Thus, it is possible to distinguish the individuals showing allegiance, who are socially valorized, and to oppose them to the rebels, devalorised. Recent studies showed that the norm of allegiance was not homogeneous and that it was possible to make a dichotomy according to "ideological" versus "mercenary" reasons conducting to the allegiance or not allegiance, this dichotomy leading to four categories (ideological allegiance, mercenary allegiance, ideological rebellion and mercenary rebellion).

In the other hand, facing a person unjustly victim of professional injustice, we can react by adopting "passive" strategies (using for example the victim's moral or behavioral responsibility: cf. Lerner), but we also can adopt "active" strategies leading to an objective re-establishment of the justice.

Our purpose was to study the impact of the allegiance on the passive $v s$ active reactions to professional injustices by examining in a differentiated way the ideological versus mercenary reasons of this allegiance or not allegiance. Our results show that the global as well as the ideological or mercenary allegiance lead to more passivity. We also observe a gender effect (men and women use different strategies) and a statute effect with a differenciation between employees and job seekers.

Key words: norm of allegiance, just world, professional injustices, passive and active reactions.

\section{Résumé}

De nombreuses études ont mis en évidence l'existence d'une norme d'allégeance que nous pouvons succinctement définir comme la valorisation sociale des individus évitant toute conduite de remise en cause de l'environnement social, et notamment, de toute remise en cause de la hiérarchie des pouvoirs dans un système social donné. Il est ainsi possible de distinguer des individus faisant preuve d'allégeance, qui sont socialement valorisés, et de les opposer aux rebelles, dévalorisés. Des études récentes ont montré que la norme d'allégeance n'était pas un tout homogène et qu'il était possible d'y effectuer, en fonction des raisons conduisant à l'allégeance ou à la non allégeance, une dichotomie selon un critère „idéologique” versus „,mercenaire”, cette dichotomie permettant d'obtenir quatre profils (allégeant idéologique, allégeant mercenaire, rebelle idéologique, rebelle mercenaire).

Sur un autre plan, face à un individu victime d'une injustice professionnelle, nous pouvons réagir en adoptant des stratégies de rééquilibrage passives (par exemple en considérant la victime responsable de son sort, comme on l'observe dans les travaux de Lerner, en évoquant une hypothétique compensation future...), mais on peut également adopter des stratégies plus ,actives” susceptibles d'aboutir à un rétablissement objectif de la justice.

L'objet du présent travail a été d'étudier l'impact de l'allégeance sur les réactions passives $v s$ actives aux injustices professionnelles en y examinant de manière différenciée les raisons idéologiques versus mercenaires de cette allégeance ou non allégeance.

Opérationnellement, un questionnaire d'allégeance de 12 items suivi de trois scénarios relatant des injustices professionnelles a été administré à 171 salariés (salariés en poste ou chômeurs) qui devaient indiquer quelles conduites (certaines passives, d'autres actives) ils préconisaient en réponse à ces injustices. Nos résultats indiquent que l'allégeance globale, idéologique et mercenaire conduisent à davantage de passivité. Nous observons égale-

${ }^{1}$ Département de Psychologie, Université de Rouen (France)

Courrier électronique: soudan.coralie@wanadoo.fr.

${ }^{2}$ Département de Psychologie, Université de Rouen (France)

Courrier électronique: bernard.gangloff@univ-rouen.fr. 
ment un effet genre (les hommes et les femmes utilisant des stratégies différentes) et, mais dans une moindre mesure, un effet statut permettant de différencier salariés en poste et chômeurs.

Mots clés: norme d'allégeance, monde juste, injustices professionnelles, réactions passives et actives.

\section{Introduction}

La vie quotidienne conduit chacun de nous à croiser, plus ou moins régulièrement, des hommes et des femmes confrontés à des situations objectivement injustes; qu'il s'agisse d'un voisin, d'un ami, ou même d'un membre de notre famille. Or selon Lerner (1965) les individus ont besoin de croire que les gens vivent dans un monde juste. La croyance en un monde juste a ainsi été définie comme la tendance à considérer que „les gens obtiennent ce qu'ils méritent et méritent ce qu'ils obtiennent" (Lerner et Simmons, 1966, p. 204), c'est-à-dire à considérer que le monde est, globalement, un lieu de justice. Aussi, face à une injustice, la plupart d'entre nous éprouve un sentiment de malaise, voire un état de dissonance cognitive (Festinger, 1957) conduisant, si la justice ne semble pas pouvoir être objectivement rétablie, à envisager des moyens permettant de la rétablir cognitivement. Les travaux de Lerner (1980), notamment, ont alors montré que, face à un individu injustement victime d'événements négatifs, nous nions fréquemment l'existence de l'injustice quitte, pour cela, à responsabiliser la victime (Lerner, 1980) ou à évoquer la malchance (Rotter, 1960) contre laquelle on ne peut rien. Pour autant, si certains individus restent ainsi passifs face aux injustices (en responsabilisant la victime, en faisant appel à un hypothétique destin qui compenserait le préjudice actuel,...), d'autres décident d'adopter des stratégies plus actives susceptibles de conduire à un rétablissement objectif de la justice*. Or si la source à l'origine de l'injustice est une source sociale (par exemple, en milieu professionnel, un supérieur hiérarchique), cet activisme conduit nécessairement à contester l'autorité de cette source.

De nombreuses études ont par ailleurs mis en évidence l'existence d'une norme d'allégeance définie comme la valorisation sociale des individus qui, dans leurs attitudes, conduites et comportements, excluent tout questionnement et donc toute remise en cause de l'environnement social, et notamment toute remise en cause de la hiérarchie des pouvoirs inhérente à cet environnement ( $c f$. pour une revue: Gangloff, 2010). Et il a été observé que si des injustices avaient pour source l'environnement social, les sujets adhérant à cette norme d'allégeance étaient peu enclins à contester les injustices constatées (Gangloff, 2005, 2006, 2007).

Des études récentes (Gangloff et Duchon, 2007) ont cependant montré que la norme d'allégeance n'était pas un tout unidimensionnel et qu'il était possible d'y effectuer, en fonction des raisons conduisant à l'adoption d'une conduite allégeante ou non-allégeante, une dichotomie selon un critère ,idéologique"(la conduite est adoptée pour défendre des valeurs) versus „,mercenaire" (on agit par arrivisme, par intérêt personnel), cette dichotomie permettant d'obtenir quatre profils: allégeant idéologique, allégeant mercenaire, rebelle idéologique, rebelle mercenaire.

Aussi avons-nous voulu examiner ici l'impact de l'allégeance avec ses sous dimensions idéologique et mercenaire quant à l'utilisation de stratégies réactionnelles passives ou actives face à des injustices professionnelles. Secondairement, nous nous sommes aussi demandé si le genre et le statut (salarié vs chômeur) avaient une influence sur les stratégies choisies.

\section{Méthode}

Nous avons demandé à 171 salariés du secteur privé de répondre successivement à deux questionnaires: un questionnaire d'allégeance puis un questionnaire présentant trois cas d'injustices professionnelles avec, à la suite de chaque cas, plusieurs réactions possibles, les unes passives et les autres traduisant des con-

* Voir par exemple, mais dans un cadre différent de celui que abordons ici, les stratégies proposées par Adams (1965) dans ses travaux sur la justive distributive. 
duites actives susceptibles de conduire à un rétablissement objectif de la justice.

L'échelle d'allégeance est constituée de douze items noyés parmi 64 questions (soit un total de 76 questions). Six items réfèrent à un comportement allégeant et six à un comportement rebelle. Ces comportements allégeants ou rebelles sont de deux sortes: idéologiques (le comportement mis en scène renvoie à une conduite que l'on peut adopter par principe, c'est-àdire pour des raisons idéologiques, pour défendre des valeurs, des convictions) ou mercenaires (c'est-à-dire pour des raisons arrivistes, par intérêt personnel). Nous avons ainsi trois items référant à de l'allégeance idéologique, trois items référant à de l'allégeance rebelle, trois items référant à de la rébellion idéologique, et trois items référant à de la rébellion mercenaire. Par exemple: „Je ne tente jamais de défendre mes idées quand je vois qu'elles sont différentes de celles de mon chef: le chef c'est le chef!” (allégeance idéologique), „quand je ne suis pas d'accord avec ce que pense mon chef, je le lui fais toujours savoir: la loi donne aussi des droits aux salariés, il faut s'en servir, sinon ils seront perdus!" (rébellion idéologique), „,comme je veux être bien vu de mon chef, j'adhère toujours à ses décisions, quelles que soient ces décisions!” (allégeance mercenaire), „quand je vois que les décisions de mon chef risquent de m'empêcher d'avoir une promotion, je n'hésite jamais à protester!" (rébellion mercenaire).

Les répondants étant invités à cocher, face à chacun des items, „d'accord” ou „pas d'accord”, nous avons attribué un point pour toute réponse allant dans le sens de l'allégeance, et 0 point dans le cas inverse. Pour la dimension allégeance prise globalement, nous obtenons ainsi une étendue allant de 0 (pas du tout allégeant, c'est-à-dire rebelle) à 12 (totalement allégeant). Pour la sous dimension idéologie, la cotation s'échelonne de 0 (pas du tout allégeant, c'est-à-dire rebelle idéologue) à 6 (totalement allégeant). Et la dimension mercenaire va aussi de 0 (pas du tout allégeant, c'est-à-dire rebelle mercenaire) à 6 (totalement allégeant) .

Le questionnaire de réactions face aux injustices professionnelles est constitué de trois scénarios ( $c f$. annexe 2): deux cas de népotisme $^{* *}$ se traduisant soit par une non embauche (cas 1) soit par un licenciement (cas 3), et un cas de licenciement par manque de souplesse (c'està-dire en fait par manque d'allégeance) de la part de la victime (cas 2).

Faisant suite à chacune des trois scénarios, neuf questions étaient présentées: la première question était destinée à vérifier que le scénario proposé était bien considéré comme injuste; les huit autres items proposaient cinq conduites de passivité et trois conduites d'activisme ${ }^{\star * \star}$. Les cinq items concernant les conduites passives étaient: 1) la responsabilisation morale de la victime (c'est la personnalité de la victime qui explique ce qui lui est arrivé), 2) la responsabilisation comportementale de la victime (la victime a eu un comportement fautif), 3) l'évocation d'un futur compensant l'injustice (il s'agit d'une minimisation de l'aspect négatif de la situation en considérant celle-ci comme très éphémère: tout va bientôt rentrer dans l'ordre), 4) l'évocation d'un futur favorisant la victime (il s'agit d'un renversement, par évocation d'un futur positif, de l'aspect négatif de la situation actuelle: la victime constatera plus tard que c'est bien mieux ainsi), 5) l'évocation d'une impuissance historique ${ }^{\star * * *}$ (est évoqué ici le fait que,

\footnotetext{
* Précisons que nous avons, sur nos 171 sujets, vérifié la consistance de l'échelle. Considérant que cette échelle est dichotomique (avec 1 ,, plutôt d'accord” et 0 ,plutôt pas d'accord” pour évaluer le degré d'approbation des sujets interrogés), nous avons privilégié le Kuder-Richardson (KR-20) plutôt qu'un alpha de Cronbach. Les analyses statistiques nous montrent alors que la consistance de l'échelle, prise globalement, est acceptable $(\operatorname{KR} 20=.78 ; F(1,168)=56.648, p$ ? 0.000). Par ailleurs, on obtient aussi des indices acceptables sur la dimension ,allégeance” (avec un Kuder-Richardson de 0.82), et sur la dimension „idéologie” (KR20 = .70). Enfin, si les dimensions non-allégeance et mercenaire présentent un Kuder-Richardson de .60 et de .56 , ces indices restent cependant significatifs ( $c f$. annexe 1$)$.

** C'est-à-dire deux cas traduisant la problématique de la discrimination étudiée notamment par Steiner ( $c f$. par exemple Steiner et Bertolino, 2006).

${ }^{* * \star}$ La littérature académique fournissant beaucoup plus d'illustrations de réactions passives qu'actives, nous avons, ici également, une non équivalence quantitative des deux types de réactions. Nous avons donc traité nos données en en tenant compte.

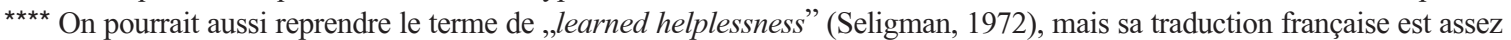
variable selon les auteurs (,résignation acquise”, ,impuissance apprise”, „,impuissance acquise”. etc.). Par ailleurs, nous ne prétendons pas que le terme „d'impuissance historique”, que nous avons choisi, réponde strictement au concept employé par Seligman.
} 
Tableau 1. Définition de chacune des propositions réactives avec leur abréviation

\begin{tabular}{|c|c|c|}
\hline Abréviation & Dénomination & Définition \\
\hline RM & Responsabilité morale & C'est la personnalité de la victime qui explique ce qui lui est arrivé \\
\hline $\mathrm{RC}$ & Responsabilité comportementale & La victime a eu un comportement fautif \\
\hline FC & Futur compensateur & $\begin{array}{l}\text { Il s'agit d'une minimisation de l'aspect négatif de la situation en considérant } \\
\text { celle-ci comme très éphémère }\end{array}$ \\
\hline FFV & Futur favorisant la victime & $\begin{array}{l}\text { II s'agit d'un renversement, par évocation d'un futur positif, de l'aspect négatif } \\
\text { de la situation actuelle }\end{array}$ \\
\hline EIH & Évocation d'une impuissance historique & Est évoqué ici le fait que, dans un cas comme celui-là, on est toujours impuissant \\
\hline $\mathrm{CCL}$ & Action de contestation collective & La victime fait appel à une association, à un syndicat \\
\hline CJا & Action de contestation juridique et individuelle & La victime entame une procédure légale afin de défendre ses droits \\
\hline $\mathrm{CIHN}$ & Action de contestation individuelle hors norme & $\begin{array}{l}\text { La victime agit de façon contestataire en adoptant des comportements } \\
\text { habituellement considérés comme étant hors normes }\end{array}$ \\
\hline
\end{tabular}

dans un cas comme celui là, on est toujours impuissant). Quant aux conduites actives, il s'agissait d'une action de contestation collective légale, d'une action de contestation judiciaire individuelle (donc légale) et enfin d'une action de contestation individuelle mais hors normes.

Le tableau 1 synthétise la définition de chacune de ces huit propositions réactionnelles (avec les abréviations que nous utiliserons ultérieurement).

A chacune des huit propositions, les sujets devaient répondre en choisissant une réponse parmi quatre (tout à fait d'accord, d'accord, pas d'accord, pas du tout d'accord), ces quatre possibilités étant ensuite, au niveau du traitement, regroupées en deux: d'accord/pas d'accord. La cotation des réponses s'est faite selon le principe suivant: pour les conduites passives il fut attribué 1 point à la réponse „d'accord” et 0 point pour „pas d'accord”; inversement, pour les conduites actives il fut attribué 0 point pour ,d'accord” et 1 point pour la réponse ,pas d'accord”

Nous obtenons ainsi: pour chaque type de conduite passive et pour l'ensemble des trois scénarios, un indice de passivité s'échelonnant de 0 (pas du tout passif) à 3 (totalement passif); et pour chaque type de conduite active et pour l'ensemble des trois scénarios, un indice d'activisme allant de 0 (totalement actif) à 3 (pas du tout actif).

Notre échantillon de départ était constitué de 171 sujets répartis en 4 groupes selon leur genre et statut professionnel: 88 hommes (dont 48 salariés en activité et 40 chômeurs) et 83 femmes (45 salariées en activité, 38 chômeuses). Mais signalons tout de suite que, compte tenu de notre objectif principal (étudier l'effet de l'allégeance), nous avons scindé notre population en deux groupes (les sujets les plus allégeants et les sujets les moins allégeants) en supprimant tous les sujets se situant dans la médiane au questionnaire d'allégeance. Nous obtenons ainsi, pour l'allégeance globale (médiane $=4) 113$ sujets répartis de la façon suivante: 51 hommes (dont 25 salariés et 26 chômeurs) et 62 femmes (dont 34 salariées et 28 chômeuses). Pour la dimension allégeance idéologique (médiane $=2$ ), notre échantillon est

Tableau 2. Répartition des sujets selon leur degré d'allégeance

\begin{tabular}{|c|c|c|c|c|c|c|c|}
\hline & & & iane (i.e. r $\mathrm{r}$ & & Sup & ane (i.e. al & \\
\hline & & Hommes & Femmes & Total & Hommes & Femmes & Total \\
\hline Allégeance & Salariés & 19 & 19 & 38 & 8 & 11 & 19 \\
\hline idéologique & Chômeurs & 16 & 16 & 32 & 8 & 11 & 19 \\
\hline (N=108) & Total & 35 & 35 & 70 & 16 & 22 & 38 \\
\hline Allégeance & Salariés & 14 & 19 & 33 & 7 & 6 & 13 \\
\hline mercenaire & Chômeurs & 13 & 15 & 28 & 8 & 7 & 15 \\
\hline$(\mathrm{N}=89)$ & Total & 27 & 34 & 61 & 15 & 13 & 28 \\
\hline Allégeance & Salariés & 12 & 15 & 27 & 13 & 19 & 32 \\
\hline globale & Chômeurs & 13 & 15 & 28 & 13 & 13 & 26 \\
\hline$(\mathrm{N}=113)$ & Total & 25 & 30 & 55 & 26 & 32 & 58 \\
\hline
\end{tabular}


de 108 sujets $(27$ hommes salariés, 24 hommes chômeurs, 30 femmes salariées et 27 femmes chômeuses). Et pour la dimension allégeance mercenaire (médiane $=3$ ), nous avons travaillé avec 89 sujets $(21$ hommes salariés, 21 hommes chômeurs, 25 femmes salariées et 22 femmes chômeuses). La répartition de nos sujets de part et d'autre de ces médianes est consignée dans le tableau 2. Enfin nous avons également supprimé tout sujet ayant considéré au moins une fois que l'un des trois cas présentés était juste (sauf lors des trois analyses ayant pour but d'examiner l'effet de cette perception de justice/injustice.

\section{Résultats*}

3.1. Effets de l'allégeance considérée globalement (i.e. en regroupant l'allégeance idéologique et l'allégeance mercenaire) et après exclusion des sujets ayant une note d'allégeance globale correspondant à la médiane (ici de 4).

Nous avons tout d'abord voulu examiner l'effet du sentiment de justice/injustice sur les stratégies choisies. Nous observons alors (tableau 3) que lorsque les sujets considèrent qu'il n'y a pas d'injustice (réponse, ,juste" aux questions $\mathrm{n}^{\circ} 1$ ), ils utilisent en moyenne plus de stratégies passives telles que la responsabilité morale et la responsabilité comportementale, et sont également, assez curieusement, davantage dans l'action contestataire individuelle légale et illégale (de même qu'ils ont tendance à plus utiliser la contestation collective: $p=0,05$ ), alors que ceux qui considèrent les situations comme injustes utilisent davantage le futur favorisant la victime.
Rappelons maintenant que les analyses suivantes ont été réalisées en supprimant tous les sujets ayant au moins une fois répondu ,juste" à la première question.

Si l'on se centre sur l'effet de l'allégeance (tableau 4), on constate tout d'abord, si nous ne tenons compte ni du genre ni du statut, que les sujets allégeants ne se distinguent pas des rebelles, même si les premiers ont tendance à plus utiliser la responsabilité comportementale que les seconds $(p=0,08)$. Chez les hommes salariés, la seule différence significative entre les allégeants et les rebelles consiste en l'utilisation de la stratégie du futur favorisant la victime qui indique que les allégeants considèrent que la victime trouvera certainement un happy end prochainement. Chez les hommes chômeurs, les stratégies de responsabilité morale, responsabilité comportementale et évocation d'une impuissance historique différencient significativement les allégeants des rebelles, les premiers utilisant davantage ces trois stratégies passives que les seconds. Chez les femmes salariées, nous constatons, assez curieusement, que les rebelles utilisent davantage que les allégeantes le futur favorisant la victime; nous remarquons aussi que, de manière tendancielle, les rebelles utilisent davantage le futur compensateur $(p=0,09)$ et moins les contestations individuelles hors normes $(p=0,08)$. Quant aux femmes chômeuses, elles se distinguent seulement par la responsabilité morale, les allégeantes étant plus enclines à l'employer que les rebelles.

Les analyses concernant l'effet du genre sont consignées au tableau 5. Sur un plan global (c'est-à-dire sans tenir compte ni du niveau d'allégeance ni du statut), on remarque (8) que les hommes sont en moyenne davantage que les femmes enclins à rendre la victime moralement

Tableau 3. Moyennes obtenues concernant les effets du sentiment de justice quels que soient le genre, le statut professionnel, et l'allégeance (les différences significatives sont indiquées en gras)

\begin{tabular}{ccccccccc}
\hline & RM & RC & FC & FFV & EIH & CCL & CJI & CIHN \\
\hline Sentiment juste & 1.594 & 1.687 & 1.375 & 1 & 1.594 & 2.312 & 2.062 & 0.844 \\
Sentiment injuste & 1.018 & 0.841 & 1.230 & 1.345 & 1.434 & 2.557 & 2.469 & 1.372 \\
$\boldsymbol{P}$ & $\mathbf{0 . 0 0 0}$ & $\mathbf{0 . 0 0 0}$ & 0.224 & $\mathbf{0 . 0 4 8}$ & 0.219 & 0.059 & $\mathbf{0 . 0 0 7}$ & $\mathbf{0 . 0 0 5}$ \\
\hline
\end{tabular}

${ }^{*}$ Les analyses ont été réalisées par des $t$ de student. 
Impact de l’allégeance en ses dimensions iddéologique et mercenaire sur les réactions aux injustices professionnelles

Tableau 4. Moyennes obtenues concernant les effets de l'allégeance globale (les différences significatives sont indiquées en gras)

\begin{tabular}{cccccccccc}
\hline & & RM & RC & FC & FFV & EIH & CCL & CJI & CIHN \\
\hline \multirow{2}{*}{ Genre et statut groupés } & Allégeant & 1.121 & 0.983 & 1.190 & 1.328 & 1.159 & 2.569 & 2.397 & 1.241 \\
& Rebelle & 0.909 & 0.691 & 1.273 & 1.364 & 1.291 & 2.545 & 2.545 & 1.509 \\
& & 0.138 & 0.082 & 0.632 & 0.853 & 0.164 & 0.873 & 0.326 & 0.168 \\
\hline \multirow{2}{*}{ Hommes salariés } & Allégeant & 1.308 & 0.923 & 1.385 & 1.769 & 1.077 & 2.462 & 2.231 & 1.538 \\
& Rebelle & 1.667 & 1.250 & 1.333 & 0.750 & 0.917 & 2.250 & 2.5 & 1.333 \\
& & 0.126 & 0.208 & 0.450 & 0.000 & 0.349 & 0.295 & 0.255 & 0.302 \\
\hline \multirow{2}{*}{ Hommes chômeurs } & Allégeant & 1.308 & 1.462 & 1.231 & 1.462 & 2.077 & 2.462 & 2.538 & 1.154 \\
& Rebelle & 0.692 & 0.462 & 1 & 1.538 & 1.231 & 2.462 & 2.692 & 1.462 \\
& & 0.031 & $\mathbf{0 . 0 0 4}$ & 0.274 & 0.434 & $\mathbf{0 . 0 1 5}$ & 0.500 & 0.275 & 0.233 \\
\hline \multirow{2}{*}{ Femmes salariées } & Allégeant & 0.895 & 0.895 & 0.947 & 1.105 & 1.421 & 2.526 & 1.474 & 1.158 \\
& Rebelle & 0.733 & 0.733 & 1.333 & 1.867 & 1.467 & 2.733 & 2.6 & 1.667 \\
& & 0.248 & 0.299 & 0.095 & 0.028 & 0.456 & 0.236 & 0.306 & 0.081 \\
\hline \multirow{2}{*}{ Femmes chômeuses } & Allégeant & 1.077 & 0.692 & 1.308 & 1.077 & 1.729 & 2.846 & 2.308 & 1.154 \\
& Rebelle & 0.667 & 0.4 & 1.4 & 1.2 & 1.467 & 2.667 & 2.4 & 1.533 \\
& & $\mathbf{0 . 0 3 3}$ & 0.137 & 0.398 & 0.351 & 0.207 & 0.185 & 0.393 & 0.185 \\
\hline
\end{tabular}

responsable de l'injustice professionnelle qu'elle subit, phénomène que l'on retrouve sur le plan de la responsabilité comportementale de manière tendancielle: $\mathrm{p}=0,05)$; par ailleurs, les femmes ont tendance $(p=0,07)$ à moins utiliser la contestation collective que les hommes. Plus analytiquement, on constate, chez les salariés allégeants, que les hommes utilisent davantage que les femmes la responsabilité morale et le futur favorisant la victime. Chez les salariés rebelles les hommes utilisent davantage que les femmes la responsabilité morale (et aussi tendanciellement la responsabilité comportementale: $p=0,08$ ) mais moins le futur favorisant la victime; sur le plan des stratégies actives, nous remarquons également que les hommes ont tendance à davantage faire appel à la contestation collective $(p=0,09)$. Chez les chômeurs

Tableau 5. Moyennes obtenues concernant les effets du genre (les différences significatives sont indiquées en gras)

\begin{tabular}{|c|c|c|c|c|c|c|c|c|c|}
\hline & & RM & RC & FC & FFV & EIH & CCL & CJI & CIHN \\
\hline \multirow{2}{*}{$\begin{array}{l}\text { Allégeance et statut } \\
\text { groupés }\end{array}$} & Hommes & 1.235 & 1.020 & 1.235 & 1.392 & 1.333 & 2.412 & 2.490 & 1.373 \\
\hline & Femmes & 0.839 & 0.694 & 1.226 & 1.306 & 1.516 & 2.677 & 2.452 & 1.371 \\
\hline \multicolumn{2}{|l|}{$p$} & 0.005 & 0.053 & 0.957 & 0.660 & 0.364 & 0.071 & 0.801 & 0.994 \\
\hline \multirow{2}{*}{ Salariés allégeants } & Hommes & 1.308 & 0.923 & 1.385 & 1.769 & 1.077 & 2.462 & 2.231 & 1.538 \\
\hline & Femmes & 0.895 & 0.895 & 0.947 & 1.105 & 1.421 & 2.526 & 1.474 & 1.158 \\
\hline \multicolumn{2}{|l|}{$p$} & 0.031 & 0.464 & 0.117 & 0.038 & 0.200 & 0.417 & 0.241 & 0.140 \\
\hline \multirow{2}{*}{ Salariés rebelles } & Hommes & 1.667 & 1.250 & 1.333 & 0.750 & 0.917 & 2.250 & 2.5 & 1.333 \\
\hline & Femmes & 0.733 & 0.733 & 1.333 & 1.867 & 1.467 & 2.733 & 2.6 & 1.667 \\
\hline \multicolumn{2}{|l|}{$p$} & 0.004 & 0.089 & 0.500 & 0.002 & 0.106 & 0.094 & 0.360 & 0.212 \\
\hline \multirow{2}{*}{ Chômeurs allégeants } & Hommes & 1.308 & 1.462 & 1.231 & 1.462 & 2.077 & 2.462 & 2.538 & 1.154 \\
\hline & Femmes & 1.077 & 0.692 & 1.308 & 1.077 & 1.729 & 2.846 & 2.308 & 1.154 \\
\hline \multicolumn{2}{|l|}{$p$} & 0.222 & 0.021 & 0.425 & 0.195 & 0.206 & 0.06 & 0.252 & 0.500 \\
\hline \multirow{2}{*}{ Chômeurs rebelles } & Hommes & 0.692 & 0.462 & 1 & 1.538 & 1.231 & 2.462 & 2.692 & 1.462 \\
\hline & Femmes & 0.667 & 0.4 & 1.4 & 1.2 & 1.467 & 2.667 & 2.4 & 1.533 \\
\hline$p$ & & 0.457 & 0.402 & 0.118 & 0.179 & 0.262 & 0.222 & 0.137 & 0.438 \\
\hline
\end{tabular}


allégeants, les hommes usent en moyenne plus la responsabilité comportementale, et tendanciellement $(\mathrm{p}=0,06)$ plus l'action collective que les femmes chômeuses allégeantes. Par contre, aucune différence significative n'apparaît entre hommes et femmes chez les chômeurs rebelles.

Enfin, sur l'effet statut (tableau 6), on remarque tout d'abord, sur un plan global, que l'évocation d'une impuissance historique est tendanciellement $(p=0,06)$ plus utilisée par les chômeurs que les salariés, phénomène que l'on retrouve, mais alors avec une différence significative, chez les hommes allégeants. Maintenant si nous comparons, chez les rebelles, les hommes salariés aux hommes chômeurs, nous constatons que les salariés utilisent en moyenne plus la stratégie de responsabilité morale et responsabilité comportementale alors que les chômeurs rebelles évoquent davantage le futur favorisant la victime. Enfin, si, chez les femmes allégeantes, aucune différence n'apparaît entre les salariées et les chômeuses, par contre, chez les femmes rebelles, les salariées croient davantage en un futur favorisant la victime que les chômeuses.

En résumé de ces analyses, il apparaît tout d'abord que les stratégies diffèrent selon que la situation est perçue comme juste ou comme injuste. Maintenant, si l'on examine les effets de l'allégeance prise globalement, plusieurs points sont à retenir. Tout d'abord que les différences entre allégeants et rebelles ne s'observent qu'au niveau des stragéties passives, et que dans ce cadre les allégeants sont quasi systématiquement plus passifs que les rebelles, avec une seule exception, qui concerne les femmes salariées. Ensuite, apparaissent aussi bien un effet genre qu'un effet statut. Ainsi, sur le plan du genre, il est notamment mis en évidence que les hommes sont toujours plus passifs que les femmes, sauf dans le sous-groupe des salariés rebelles, où alors les femmes utilisent plus que les hommes le futur favorisant la victime. Quant à l'effet un effet statut, retenons seulement ici qu'il est peu fréquent et que sa direction varie notamment selon le genre.

3.2. Effets de l'allégeance idéologique après exclusion des sujets ayant une note d'allégeance idéologique

correspondant à la médiane (ici de 2).

Nous avons tout d'abord là aussi voulu examiner l'effet du sentiment de justice/injustice sur les stratégies choisies. Nous constatons alors (tableau 7) que les sujets qui ont le sentiment que la situation est juste utilisent les stratégies de responsabilité morale et comportementale et la contestation individuelle hors norme

Tableau 6. Moyennes obtenues concernant les effets du statut (les différences significatives sont indiquées en gras)

\begin{tabular}{|c|c|c|c|c|c|c|c|c|c|}
\hline & & RM & RC & FC & FFV & EIH & CCL & CJI & $\mathrm{CIHN}$ \\
\hline \multirow{3}{*}{$\begin{array}{l}\text { Allégeance et } \\
\text { genre groupés }\end{array}$} & Salariés & 1.102 & 0.932 & 1.220 & 1.373 & 1.254 & 2.508 & 2.458 & 1.407 \\
\hline & Chômeurs & 0.926 & 0.741 & 1.241 & 1.375 & 1.630 & 2.611 & 2.481 & 1.333 \\
\hline & $p$ & 0.218 & 0.256 & 0.907 & 0.765 & 0.060 & 0.486 & 0.875 & 0.706 \\
\hline \multirow{3}{*}{ Hommes allégeants } & Hommes salariés & 1.308 & 0.923 & 1.385 & 1.769 & 1.077 & 2.462 & 2.231 & 1.538 \\
\hline & Hommes chômeurs & 1.308 & 1.462 & 1.231 & 1.462 & 2.077 & 2.462 & 2.538 & 1.154 \\
\hline & $p$ & 0.500 & 0.830 & 0.367 & 0.208 & 0.002 & 0.5 & 0.197 & 0.127 \\
\hline \multirow{3}{*}{ Hommes rebelles } & Hommes salariés & 1.667 & 1.250 & 1.333 & 0.750 & 0.917 & 2.250 & 2.5 & 1.333 \\
\hline & Hommes chômeurs & 0.692 & 0.462 & 1 & 1.538 & 1.231 & 2.462 & 2.692 & 1.462 \\
\hline & $p$ & 0.003 & 0.017 & 0.154 & 0.027 & 0.247 & 0.295 & 0.270 & 0.394 \\
\hline \multirow{3}{*}{$\begin{array}{l}\text { Femmes } \\
\text { allégeantes }\end{array}$} & Femmes salariées & 0.895 & 0.895 & 0.947 & 1.105 & 1.421 & 2.526 & 1.474 & 1.158 \\
\hline & Femmes chômeuses & 1.077 & 0.692 & 1.308 & 1.077 & 1.729 & 2.846 & 2.308 & 1.154 \\
\hline & $p$ & 0.202 & 0.251 & 0.135 & 0.473 & 0.213 & 0.120 & 0.310 & 0.496 \\
\hline \multirow{3}{*}{ Femmes rebelles } & Femmes salariées & 0.733 & 0.733 & 1.333 & 1.867 & 1.467 & 2.733 & 2.6 & 1.667 \\
\hline & Femmes chômeuses & 0.667 & 0.4 & 1.4 & 1.2 & 1.467 & 2.667 & 2.4 & 1.533 \\
\hline & $p$ & 0.392 & 0.122 & 0.417 & 0.025 & 0.500 & 0.392 & 0.197 & 0.370 \\
\hline
\end{tabular}


Impact de l'allégeance en ses dimensions idéologique et mercenaire sur les réactions aux injustices professionnelles

Tableau 7. Moyennes obtenues concernant les effets du sentiment de justice quels que soient le genre, le statut professionnel et l'allégeance idéologique (les différences significatives sont indiquées en gras)

\begin{tabular}{ccccccccc}
\hline & RM & RC & FC & FFV & EIH & CCL & CJI & CIHN \\
\hline Sentiment juste & 1.613 & 1.645 & 1.161 & 0.903 & 1.516 & 2.323 & 2.129 & 0.903 \\
Sentiment injuste & 1.028 & 0.843 & 1.287 & 1.333 & 1.444 & 2.528 & 2.389 & 1.352 \\
$\boldsymbol{p}$ & $\mathbf{0 . 0 0 0}$ & $\mathbf{0 . 0 0 0}$ & 0.264 & $\mathbf{0 . 0 2 0}$ & 0.368 & 0.110 & 0.070 & $\mathbf{0 . 0 1 8}$ \\
\hline
\end{tabular}

(et tendanciellement $-\mathrm{p}=0,07$ - la contestation juridique individuelle). Par contre ceux qui perçoivent cette situation comme injuste évoquent un futur favorisant la victime dans le but de remédier objectivement à l'injustice.

Commençons par rappeler que les analyses suivantes ont été réalisées en supprimant tous les sujets ayant au moins une fois répondu ,juste" à la première question. Maintenant, au niveau des résultats globaux tout d'abord, il apparaît que les allégeants idéologiques remédient davantage aux injustices professionnelles par le biais de la responsabilité comportementale et l'évocation d'une impuissance historique que ne le font les rebelles idéologiques. Plus précisément, il est constaté, chez les hommes salariés, que le futur favorisant la victime est en moyenne plus employé par les allégeants que par les rebelles, et chez les hommes chômeurs que les allégeants font davantage usage de stratégies de respons- abilité morale, responsabilité comportementale, évocation d'une impuissance historique et (tendanciellement $-p=0,07$ - futur compensateur) que les rebelles. Quant aux femmes, chez les salariées, force est de constater qu'il n'existe pas de différence significative entre les allégeantes et les rebelles, à l'exception de deux tendances, les rebelles acceptant davantage le futur favorisant la victime $(p=0,06)$ et les actions collectives $(p=0,07)$. Enfin, chez les femmes au chômage, les allégeantes se distinguent des rebelles en expliquant en moyenne davantage l'injustice perçue par l'évocation d'une impuissance historique et en utilisant moins la stratégie de contestation collective.

Concernant les effets du genre (tableau 9), il apparaît, sur un plan global, que l'évocation d'une impuissance historique est davantage employée par les femmes pour contrer les injustices professionnelles, mais que par contre les

Tableau 8. Moyennes obtenues concernant les effets de l'allégeance idéologique (les différences significatives sont indiquées en gras)

\begin{tabular}{|c|c|c|c|c|c|c|c|c|c|}
\hline & & RM & RC & FC & FFV & EIH & CCL & CJI & CIHN \\
\hline \multirow{2}{*}{$\begin{array}{l}\text { Genre et statut } \\
\text { groupés }\end{array}$} & Allégeant & 1.132 & 1.079 & 1.368 & 1.395 & 1.842 & 2.632 & 2.289 & 1.237 \\
\hline & Rebelle & 0.971 & 0.714 & 1.243 & 1.300 & 1.229 & 2.471 & 2.443 & 1.414 \\
\hline \multicolumn{2}{|l|}{$p$} & 0.294 & 0.048 & 0.518 & 0.645 & 0.004 & 0.345 & 0.380 & 0.409 \\
\hline \multirow{2}{*}{ Hommes salariés } & Allégeant & 1.125 & 0.750 & 1.5 & 1.875 & 1.250 & 2.250 & 2 & 1.375 \\
\hline & Rebelle & 1.474 & 1.053 & 1.316 & 0.842 & 0.842 & 2.421 & 2.368 & 1.368 \\
\hline$p$ & & 0.153 & 0.228 & 0.342 & 0.002 & 0.176 & 0.335 & 0.218 & 0.494 \\
\hline \multirow{2}{*}{ Hommes chômeurs } & Allégeant & 1.5 & 2 & 1.625 & 1.5 & 2.250 & 2.250 & 2.375 & 1 \\
\hline & Rebelle & 0.688 & 0.438 & 1 & 1.5 & 1.125 & 2.563 & 2.625 & 1.375 \\
\hline$p$ & & 0.013 & 0.000 & 0.072 & 0.500 & 0.005 & 0.183 & 0.218 & 0.224 \\
\hline \multirow{2}{*}{ Femmes salariées } & Allégeant & 0.909 & 1 & 1.091 & 1.182 & 1.818 & 2.818 & 2.545 & 1.545 \\
\hline & Rebelle & 0.789 & 0.842 & 1.263 & 1.789 & 1.737 & 2.263 & 0.368 & 1.474 \\
\hline$p$ & & 0.330 & 0.330 & 0.316 & 0.069 & 0.427 & 0.075 & 0.265 & 0.430 \\
\hline \multirow{2}{*}{ Femmes chômeuses } & Allégeant & 1.091 & 0.727 & 1.364 & 1.182 & 2 & 3 & 2.182 & 1 \\
\hline & Rebelle & 0.875 & 0.438 & 1.375 & 1.063 & 1.188 & 2.688 & 2.438 & 1.438 \\
\hline$p$ & & 0.179 & 0.183 & 0.488 & 0.370 & 0.012 & 0.049 & 0.234 & 0.166 \\
\hline
\end{tabular}


Tableau 9. Moyennes obtenues concernant les effets du genre (les différences significatives sont indiquées en gras)

\begin{tabular}{|c|c|c|c|c|c|c|c|c|c|}
\hline & & RM & $\mathrm{RC}$ & FC & FFV & EIH & CCL & CJI & CIHN \\
\hline \multirow{2}{*}{$\begin{array}{l}\text { Allégeance et } \\
\text { statut groupés }\end{array}$} & Hommes & 1.176 & 0.961 & 1.294 & 1.314 & 1.216 & 2.412 & 2.392 & 1.314 \\
\hline & Femmes & 0.895 & 0.737 & 1.281 & 1.351 & 1.649 & 2.632 & 2.632 & 1.386 \\
\hline \multicolumn{2}{|l|}{$p$} & 0.052 & 0.208 & 0.942 & 0.850 & 0.035 & 0.174 & 0.971 & 0.726 \\
\hline \multirow{2}{*}{ Salariés allégeants } & Hommes & 1.125 & 0.750 & 1.5 & 1.875 & 1.250 & 2.250 & 2 & 1.375 \\
\hline & Femmes & 0.909 & 1 & 1.091 & 1.182 & 1.818 & 2.818 & 2.545 & 1.545 \\
\hline$p$ & & 0.218 & 0.290 & 0.208 & 0.062 & 0.155 & 0.057 & 0.112 & 0.349 \\
\hline \multirow{2}{*}{ Salariés rebelles } & Hommes & 1.474 & 1.053 & 1.316 & 0.842 & 0.842 & 2.421 & 2.368 & 1.368 \\
\hline & Femmes & 0.789 & 0.842 & 1.263 & 1.789 & 1.737 & 2.263 & 0.368 & 1.474 \\
\hline$p$ & & 0.007 & 0.246 & 0.434 & 0.002 & 0.007 & 0.324 & 0.500 & 0.384 \\
\hline \multirow{2}{*}{$\begin{array}{l}\text { Chômeurs } \\
\text { allégeants }\end{array}$} & Hommes & 1.5 & 2 & 1.625 & 1.5 & 2.250 & 2.250 & 2.375 & 1 \\
\hline & Femmes & 1.091 & 0.727 & 1.364 & 1.182 & 2 & 3 & 2.182 & 1 \\
\hline \multicolumn{2}{|l|}{$p$} & 0.093 & 0.001 & 0.307 & 0.276 & 0.277 & 0.001 & 0.334 & 0.500 \\
\hline \multirow{2}{*}{ Chômeurs rebelles } & Hommes & 0.688 & 0.438 & 1 & 1.5 & 1.125 & 2.563 & 2.625 & 1.375 \\
\hline & Femmes & 0.875 & 0.438 & 1.375 & 1.063 & 1.188 & 2.688 & 2.438 & 1.438 \\
\hline$p$ & & 0.231 & 0.500 & 0.111 & 0.109 & 0.432 & 0.313 & 0.235 & 0.441 \\
\hline
\end{tabular}

hommes seraient tendanciellement $(\mathrm{p}=0,05)$ plus enclins à employer la responsabilité morale que les femmes. Chez les salariés allégeants, il n'existe que deux différences tendancielles, les hommes évoquant davantage le futur favorisant la victime $(p=0,06)$ et l'action collective $(p=0,05)$ que les femmes. Chez les salariés rebelles, les hommes sont plus enclins à remédier à l'injustice perçue par la responsabilité morale tandis que les femmes préfèrent l'évocation d'un futur favorisant la victime et d'une impuissance historique. Chez les chômeurs allégeants, nous constatons que les hommes font plus appel à la responsabilité comportementale et à l'action collective (et tendanciellement à la responsabilité morale: $p=0,09$ ) que les femmes. Par contre, aucune différence entre hommes et femmes n'est constatée chez les chômeurs rebelles.

Enfin, pour les effets du statut (tableau 10), si on ne remarque, à un niveau global, aucune différence, par contre des analyses plus fines mettent en évidence plusieurs effets. Chez les hommes allégeants, les chômeurs sont en moyenne, par rapport aux salariés, plus disposés à remédier à l'injustice perçue par le biais de stratégies telles que la responsabilité morale et l'évocation d'une impuissance historique. Chez les hommes rebelles trois stratégies conduisent à des différences significatives: la responsabilité morale, et la responsabilité comportementale (favorisées par les salariés) et le futur favorisant la victime (davantage choisie par les chômeurs). Chez les femmes allégeantes les salariées et les chômeuses ne se distinguent que tendanciellement avec plus de contestations collectives proposées par les salariées $(p=0,07)$. Enfin, chez les femmes rebelles, les salariées évoquent plus que ne le font les chômeuses, un futur favorisant la victime et une impuissance historique (et tendanciellement la responsabilité comportementale: $p=0,08$ )

En résumé de ces analyses, il apparaît ici encore que les stratégies diffèrent selon que la situation est perçue comme juste ou comme injuste. L'examen des stratégies utilisées en fonction $\mathrm{du}$ niveau d'allégeance/rébellion idéologique montre essentiellement que les allégeants sont systématiquement plus passifs que les rebelles, mais ici sans aucune exception significative. Des effets genre sont cependant mis en évidence, avec notamment des hommes qui sont les seuls à mettre en avant la responsabilité morale ou comportementale de la victime, mais aussi des hommes qui sont les seuls à 
Impact de l’allégeance en ses dimensions iddéologique et mercenaire sur les réactions aux injustices professionnelles

Tableau 10. Moyennes obtenues concernant les effets du statut (les différences significatives sont indiquées en gras)

\begin{tabular}{|c|c|c|c|c|c|c|c|c|c|}
\hline & & RM & RC & FC & FFV & EIH & CCL & CJI & CIHN \\
\hline \multirow{3}{*}{$\begin{array}{l}\text { Allégeance et } \\
\text { genre groupés }\end{array}$} & Salariés & 1.088 & 0.930 & 1.281 & 1.368 & 1.386 & 2.421 & 2.351 & 1.439 \\
\hline & Chômeurs & 0.961 & 0.745 & 1.294 & 1.294 & 1.510 & 2.647 & 2.431 & 1.255 \\
\hline & $p$ & 0.385 & 0.299 & 0.942 & 0.706 & 0.551 & 0.162 & 0.631 & 0.372 \\
\hline \multirow{3}{*}{$\begin{array}{l}\text { Hommes } \\
\text { allégeants }\end{array}$} & Hommes salariés & 1.125 & 0.750 & 1.5 & 1.875 & 1.250 & 2.250 & 2 & 1.375 \\
\hline & Hommes chômeurs & 1.5 & 2 & 1.625 & 1.5 & 2.250 & 2.250 & 2.375 & 1 \\
\hline & $p$ & 0.151 & 0.005 & 0.418 & 0.224 & 0.020 & 0.500 & 0.246 & 0.194 \\
\hline \multirow{3}{*}{ Hommes rebelles } & Hommes salariés & 1.474 & 1.053 & 1.316 & 0.842 & 0.842 & 2.421 & 2.368 & 1.368 \\
\hline & Hommes chômeurs & 0.688 & 0.438 & 1 & 1.5 & 1.125 & 2.563 & 2.625 & 1.375 \\
\hline & $p$ & 0.004 & 0.018 & 0.160 & 0.030 & 0.210 & 0.316 & 0.201 & 0.493 \\
\hline \multirow{3}{*}{$\begin{array}{l}\text { Femmes } \\
\text { allégeantes }\end{array}$} & Femmes salariées & 0.909 & 1 & 1.091 & 1.182 & 1.818 & 2.818 & 2.545 & 1.545 \\
\hline & Femmes chômeuses & 1.091 & 0.727 & 1.364 & 1.182 & 2 & 3 & 2.182 & 1 \\
\hline & $p$ & 0.219 & 0.243 & 0.262 & 0.500 & 0.355 & 0.076 & 0.163 & 0.122 \\
\hline \multirow{3}{*}{ Femmes rebelles } & Femmes salariées & 0.789 & 0.842 & 1.263 & 1.789 & 1.737 & 2.263 & 0.368 & 1.474 \\
\hline & Femmes chômeuses & 0.875 & 0.438 & 1.375 & 1.063 & 1.188 & 2.688 & 2.438 & 1.438 \\
\hline & $p$ & 0.364 & 0.088 & 0.360 & 0.013 & 0.049 & 0.103 & 0.400 & 0.462 \\
\hline
\end{tabular}

parfois proposer l'emploi d'une stratégie active (en l'occurrence l'utilisation de la contestation collective légale). Enfin, sur le plan du statut, on observe que les rebelles (qu'ils soient hommes ou femmes) utilisent généralement plus de stratégies passives quand ils sont salariés que lorsqu'ils sont au chômage, l'inverse n'étant généralement observé chez les allégeants.

3.3. Effets de l'allégeance mercenaire après exclusion des sujets ayant une note d'allégeance mercenaire correspondant à la médiane (ici de 3).

Nous avons encore, là aussi, examiné l'effet du sentiment de justice/injustice sur les stratégies choisies. Il apparaît ici (tableau 11), que les sujets qui considèrent les situations présentées comme justes font plus appel à la responsabilité de la victime (tant sur le plan moral que comportemental) et également (de manière étrange) aux trois stratégies de contestation (quoique ce ne soit que tendanciellement pour les actions collectives: $p=0,09$ ).

Rappelons, qu'ici également, toutes les analyses suivantes ont été réalisées en supprimant tout sujet ayant au moins une fois répondu ,juste" à la première question.

L'examen de la variable allégance mercenaire prise globalement n'indique aucune différence significative. De même, chez les hommes salariés, nous n'observons que des tendances, les allégeants faisant davantage état du futur favorisant la victime $(p=0,05)$ et moins de l'impuissance historique $(p=0,08)$ que les rebelles. Chez les hommes au chômage, les allégeants (par rapport aux rebelles) rétablissent davantage l'injustice perçue par la responsabilité morale, par la responsabilité comportementale

Tableau 11. Moyennes obtenues concernant les effets du sentiment de justice quels que soient le genre, le statut professionnel et l'allégeance mercenaire (les différences significatives sont indiquées en gras)

\begin{tabular}{ccccccccc}
\hline & RM & RC & FC & FFV & EIH & CCL & CJI & CIHN \\
\hline Sentiment juste & 1.571 & 1.762 & 1.286 & 1.095 & 1.476 & 2.286 & 2 & 0.857 \\
Sentiment injuste & 1.022 & 0.876 & 1.146 & 1.359 & 1.404 & 2.539 & 2.438 & 1.104 \\
$\boldsymbol{p}$ & $\mathbf{0 . 0 0 3}$ & $\mathbf{0 . 0 0 0}$ & 0.281 & 0.150 & 0.389 & 0.096 & $\mathbf{0 . 0 1 2}$ & $\mathbf{0 . 0 1 3}$ \\
\hline
\end{tabular}


Impact de l'allégeance en ses dimensions idéologique et mercenaire sur les réactions aux injustices professionnelles

Tableau 12. Moyennes obtenues concernant les effets de l'allégeance mercenaire (les différences significatives sont indiquées en gras)

\begin{tabular}{|c|c|c|c|c|c|c|c|c|c|}
\hline & & RM & RC & FC & FFV & EIH & CCL & CJI & CIHN \\
\hline \multirow{2}{*}{$\begin{array}{l}\text { Genre et statut } \\
\text { groupés }\end{array}$} & Allégeant & 1.143 & 1 & 1.071 & 1.357 & 1.679 & 2.5 & 2.393 & 1.286 \\
\hline & Rebelle & 0.967 & 0.820 & 1.180 & 1.361 & 1.279 & 2.557 & 2.459 & 1.459 \\
\hline \multicolumn{2}{|l|}{$p$} & 0.337 & 0.409 & 0.626 & 0.988 & 0.107 & 0.755 & 0.718 & 0.464 \\
\hline \multirow{2}{*}{$\begin{array}{l}\text { Hommes } \\
\text { salariés }\end{array}$} & Allégeant & 1.143 & 0.714 & 1.143 & 1.571 & 0.428 & 2.571 & 2.428 & 1.714 \\
\hline & Rebelle & 1.643 & 1.286 & 1.214 & 0.928 & 1.071 & 2.143 & 2.286 & 1.5 \\
\hline \multicolumn{2}{|l|}{$p$} & 0.112 & 0.134 & 0.443 & 0.052 & 0.088 & 0.186 & 0.379 & 0.315 \\
\hline \multirow{2}{*}{$\begin{array}{l}\text { Hommes } \\
\text { chômeurs }\end{array}$} & Allégeant & 1.625 & 1.750 & 1.375 & 1.375 & 2.375 & 2.250 & 2.375 & 1 \\
\hline & Rebelle & 0.769 & 0.538 & 0.846 & 1.461 & 1.154 & 2.538 & 2.692 & 1.615 \\
\hline \multicolumn{2}{|l|}{$p$} & 0.009 & 0.001 & 0.130 & 0.439 & 0.005 & 0.221 & 0.154 & 0.115 \\
\hline \multirow{3}{*}{$\begin{array}{l}\text { Femmes } \\
\text { salariées }\end{array}$} & Allégeant & 0.833 & 0.833 & 0.667 & 1.167 & 1.833 & 2.5 & 1.833 & 1 \\
\hline & Rebelle & 0.789 & 0.895 & 1.158 & 1.737 & 1.421 & 2.737 & 2.579 & 1.579 \\
\hline & & 0.449 & 0.425 & 0.123 & 0.143 & 0.230 & 0.255 & 0.017 & 0.131 \\
\hline \multirow{2}{*}{$\begin{array}{l}\text { Femmes } \\
\text { chômeuses }\end{array}$} & Allégeant & 0.857 & 0.571 & 1 & 1.286 & 2 & 2.714 & 2.857 & 1.428 \\
\hline & Rebelle & 0.733 & 0.533 & 1.467 & 1.2 & 1.4 & 2.733 & 2.267 & 1.133 \\
\hline \multicolumn{2}{|l|}{$p$} & 0.335 & 0.457 & 0.172 & 0.422 & 0.077 & 0.471 & 0.054 & 0.270 \\
\hline
\end{tabular}

et par l'évocation d'une impuissance historique. Chez les femmes salariées maintenant, les allégeantes sont étonnamment plus actives que les rebelles en utilisant davantage que ces dernières la contestation juridique individuelle. Enfin, chez les femmes au chômage, nous observons une tendance des rebelles à utiliser davantage la contestation judiciaire individuelle $(\mathrm{p}=0,05)$ tandis que les allégeantes ont tendance à évoquer une impuissance historique $(\mathrm{p}=0.07)$.

Concernant les effets du genre maintenant (tableau 13), on constate que, globalement, les hommes s'avèrent plus passifs que les femmes, en utilisant davantage que ces dernières la

Tableau 13. Moyennes obtenues concernant les effets du genre (les différences significatives sont indiquées en gras)

\begin{tabular}{|c|c|c|c|c|c|c|c|c|c|}
\hline & & RM & RC & FC & FFV & EIH & CCL & CJI & CIHN \\
\hline \multirow{2}{*}{$\begin{array}{l}\text { Allégeance et } \\
\text { statut groupés }\end{array}$} & Hommes & 1.286 & 1.048 & 1.119 & 1.286 & 1.238 & 2.357 & 2.452 & 1.476 \\
\hline & Femmes & 0.787 & 0.723 & 1.170 & 1.426 & 1.553 & 2.702 & 2.426 & 1.340 \\
\hline \multicolumn{2}{|c|}{$p$} & 0.003 & 0.109 & 0.806 & 0.528 & 0.172 & 0.041 & 0.875 & 0.538 \\
\hline \multirow{2}{*}{$\begin{array}{c}\text { Salariés } \\
\text { allégeants }\end{array}$} & Hommes & 1.143 & 0.714 & 1.143 & 1.571 & 0.428 & 2.571 & 2.428 & 1.714 \\
\hline & Femmes & 0.833 & 0.833 & 0.667 & 1.167 & 1.833 & 2.5 & 1.833 & 1 \\
\hline \multicolumn{2}{|c|}{$p$} & 0.228 & 0.405 & 0.248 & 0.236 & 0.019 & 0.428 & 0.186 & 0.117 \\
\hline \multirow{2}{*}{ Salariés rebelles } & Hommes & 1.643 & 1.286 & 1.214 & 0.928 & 1.071 & 2.143 & 2.286 & 1.5 \\
\hline & Femmes & 0.789 & 0.895 & 1.158 & 1.737 & 1.421 & 2.737 & 2.579 & 1.579 \\
\hline \multicolumn{2}{|c|}{$p$} & 0.003 & 0.151 & 0.427 & 0.014 & 0.187 & 0.041 & 0.124 & 0.414 \\
\hline \multirow{3}{*}{$\begin{array}{l}\text { Chômeurs } \\
\text { allégeants }\end{array}$} & Hommes & 1.625 & 1.750 & 1.375 & 1.375 & 2.375 & 2.250 & 2.375 & 1 \\
\hline & Femmes & 0.857 & 0.571 & 1 & 1.286 & 2 & 2.714 & 2.857 & 1.428 \\
\hline & & 0.030 & 0.015 & 0.274 & 0.445 & 0.184 & 0.120 & 0.073 & 0.217 \\
\hline \multirow{3}{*}{$\begin{array}{l}\text { Chômeurs } \\
\text { rebelles }\end{array}$} & Hommes & 0.769 & 0.538 & 0.846 & 1.461 & 1.154 & 2.538 & 2.692 & 1.615 \\
\hline & Femmes & 0.733 & 0.533 & 1.467 & 1.2 & 1.4 & 2.733 & 2.267 & 1.133 \\
\hline & $p$ & 0.443 & 0.492 & 0.041 & 0.254 & 0.258 & 0.229 & 0.080 & 0.127 \\
\hline
\end{tabular}


Tableau 14. Moyennes obtenues concernant les effets du statut (les différences significatives sont indiquées en gras)

\begin{tabular}{|c|c|c|c|c|c|c|c|c|c|}
\hline & & RM & RC & FC & FFV & EIH & CCL & CJI & CIHN \\
\hline \multirow{3}{*}{$\begin{array}{l}\text { Allégeance et } \\
\text { genre groupés }\end{array}$} & Salariés & 1.109 & 0.978 & 1.109 & 1.391 & 1.217 & 2.5 & 2.370 & 1.5 \\
\hline & Chômeurs & 0.930 & 0.737 & 1.186 & 1.326 & 1.605 & 2.581 & 2.512 & 1.302 \\
\hline & $p$ & 0.294 & 0.299 & 0.740 & 0.767 & 0.092 & 0.634 & 0.404 & 0.369 \\
\hline \multirow{3}{*}{$\begin{array}{l}\text { Hommes } \\
\text { allégeants }\end{array}$} & Hommes salariés & 1.143 & 0.714 & 1.143 & 1.571 & 0.428 & 2.571 & 2.428 & 1.714 \\
\hline & Hommes chômeurs & 1.625 & 1.750 & 1.375 & 1.375 & 2.375 & 2.250 & 2.375 & 1 \\
\hline & $p$ & 0.138 & 0.033 & 0.375 & 0.367 & 0.000 & 0.172 & 0.457 & 0.082 \\
\hline \multirow{3}{*}{ Hommes rebelles } & Hommes salariés & 1.643 & 1.286 & 1.214 & 0.928 & 1.071 & 2.143 & 2.286 & 1.5 \\
\hline & Hommes chômeurs & 0.769 & 0.538 & 0.846 & 1.461 & 1.154 & 2.538 & 2.692 & 1.615 \\
\hline & $p$ & 0.004 & 0.025 & 0.122 & 0.094 & 0.424 & 0.173 & 0.097 & 0.391 \\
\hline \multirow{3}{*}{$\begin{array}{l}\text { Femmes } \\
\text { allégeantes }\end{array}$} & Femmes salariées & 0.833 & 0.833 & 0.667 & 1.167 & 1.833 & 2.5 & 1.833 & 1 \\
\hline & Femmes chômeuses & 0.857 & 0.571 & 1 & 1.286 & 2 & 2.714 & 2.857 & 1.428 \\
\hline & $p$ & 0.471 & 0.277 & 0.264 & 0.427 & 0.401 & 0.318 & 0.025 & 0.252 \\
\hline \multirow{3}{*}{ Femmes rebelles } & Femmes salariées & 0.789 & 0.895 & 1.158 & 1.737 & 1.421 & 2.737 & 2.579 & 1.579 \\
\hline & Femmes chômeuses & 0.733 & 0.533 & 1.467 & 1.2 & 1.4 & 2.733 & 2.267 & 1.133 \\
\hline & $p$ & 0.410 & 0.125 & 0.174 & 0.065 & 0.476 & 0.494 & 0.102 & 0.111 \\
\hline
\end{tabular}

responsabilité morale, par contre ils sont plus dans contestation collective que ne le sont les femmes. Chez les salariés allégeants, on remarque que les femmes évoquent plus fréquemment l'impuissance historique que ne le font les hommes. Chez les salariés rebelles, on constate, d'une part que les hommes sont en moyenne plus passifs que les femmes en utilisant davantage la responsabilité morale et plus contestataires par le biais de la contestation collective légale, mais d'autre part que les femmes sont en moyenne plus favorables à l'usage de stratégies favorisant la victime (futur favorisant la victime). Chez les chômeurs allégeants, les hommes sont en moyenne plus passifs que les femmes par le biais de la responsabilité (comportementale et morale) mais ont une tendance à davantage proposer des contestations juridiques individuelles $(p=0,07)$. Enfin, chez les chômeurs rebelles, on peut remarquer que les femmes emploient plus que les hommes le futur compensateur et (tendanciellement) les actions juridiques individuelles $(\mathrm{p}=0,08)$.

Maintenant, sur les effets du statut (tableau 14), et tout d'abord de manière globale, même si l'on constate l'absence de toute différence significative, il est à noter que, tendanciellement, les chômeurs évoquent davantage une impuissance historique que les salariés $(\mathrm{p}=0.09)$. Chez les hommes allégeants, il apparaît que les chômeurs sont plus passifs que les salariés en utilisant plus que ces derniers la responsabilité comportementale et l'impuissance historique, par contre, ils auraient aussi tendance à employer davantage la contestation individuelle hors norme $(p=0,08)$. Chez les hommes rebelles, les salariés, comparativement aux chômeurs, emploient plus de stratégies de remédiation telles que la responsabilité (comportementale et morale) pour rétablir la justice, et tendanciellement aussi davantage la contestation juridique individuelle $(p=0,09)$ et moins le futur favorisant la victime $(p=0,09)$. Chez les femmes allégeantes les salariées pallient davantage les injustices professionnelles par le biais de la contestation juridique individuelle que ne le font les femmes au chômage. Enfin, chez les femmes rebelles nous observons une tendance des salariées rebelles à préférer davantage le futur favorisant la victime que les chômeuses $(\mathrm{p}=0,06)$.

En synthèse de ces derniers résultats, nous noterons tout d'abord que la perception de la situation comme étant juste ou injuste influence à nouveau le choix des stratégies à privilégier. Par ailleurs, nous remarquons encore une fois que les allégeants sont toujours significativement plus 
passifs que les rebelles; pour autant, une exception apparaît sur le plan des stratégies actives, avec des femmes salariées allégeantes prônant davantage la contestation juridique individuelle que ne le font les femmes salariées rebelles. On observe aussi un effet genre, avec des hommes qui sont le seuls à employer la responsabilité morale ou comportementale, et a contrario une utilisation du futur compensateur, du futur favorisant la victime et de l'impuissance historique qui ne s'observent que chez les femmes. Parallèlement à cela, les hommes sont les seuls à proposer la contestation collective légale. Sur le plan de l'effet statut, on remarque essentiellement que les chômeurs utilisent moins de stratégies passives que les salariés, sauf dans la souspopulation des hommes allégeants.

\section{Conclusion}

Le but de cette recherche était d'examiner quelques-unes des stratégies potentielles de remédiation aux injustices professionnelles, et notamment d'étudier leur utilisation différenciée selon le niveau d'allégeance du répondant, que cette allégeance soit considérée de manière globale ou sur la base de chacun de ses deux fondements: fondement idéologique et fondement mercenaire. Nous avons, pour cela, commencé par vérifier que les cas que nous présentions étaient bien perçus comme injustes. Constatant cependant que ce n'était pas toujours le cas, nous avons d'abord décidé d'utiliser cette perception différenciée comme une variable indépendante et d'examiner son éventuelle influence sur le choix des conduites réactives proposées. Nous avons alors constaté que cette perception différenciée conduisait effectivement à des préférences stratégiques différentes, et notamment que l'on pouvait à la fois opter pour des réactions de passivité et en même temps considérer qu'il était souhaitable d'adopter une conduite active permettant de normaliser la situation. Que ce soit sur le plan de l'allégeance globale ou en ses différenciations idéologique/mercenaire, il a en effet été constaté que les répondants qui considéraient les situations comme justes étaient les plus enclins à évoquer la responsabilité morale ou comportementale de la victime, mais également que cétait ces mêmes répondants qui proposaient de porter plainte (contestation juridique individuelle), voire même de réagir de manière hors normes. Il est donc retrouvé ici certains des constats mis en évidence dans le cadre des études sur le monde juste lorsqu'il est obsservé, dans des cas d'agression, que l'on peut à la fois juger la victime partiellement responsable de sa mésaventure et dans le même temps souhaiter que l'agresseur soit condamné ( $c f$. par exemple Jones et Aronson, 1973; ou encore Kenrick, Reich et Cialdini, 1976). Plus globalement, ces résultats ont aussi permis de mettre en évidence que si la responsabilité morale et la responsabilité comportementale sont effectivement employées, d'autres réactions, comme l'évocation d'une impuissance historique, un futur favorisant la victime,... ne sont pas à négliger.

En ce qui trait à notre problématique principale, c'est-à-dire à l'influence de l'allégeance, plusieurs points sont à retenir. Tout d'abord, en considérant l'allégeance globalement, on remarque que les différences entre allégeants et rebelles ne s'observent qu'au niveau des stragéties passives, et que dans ce cadre les allégeants utilisent quasi systématiquement plus de stratégies passives que les rebelles, avec une seule exception, qui concerne les femmes salariées. Le même constat peut être réalisé si l'on se centre sur l'allégeance idéologique, mais ici sans aucune exception significative. Enfin, au niveau de l'allégeance mercenaire, si les résultats vont dans le même sens au niveau des stratégies passives, on contate par contre que des différences se font maintenant aussi jour sur les stratégies actives, avec des femmes salariées allégeantes prônant davantage la contestation juridique individuelle que ne le font les femmes salariées rebelles. Si la plupart de ces résultats sont tout à fait logiques, et attestent de l'intérêt d'appronfondir la différenciation allégeance idéologqiue/allégeance mercenaire qui conduit à des réactions non systématiquementy identiques, par contre le dernier résultat, sur l'utilisation des stragégies actives, nous semble quelque peu contre-intuitif. Nous n'avons cependant pas, en l'état actuel de nos données, d'éléments suffisants permettant de l'expliquer. 
Nous souhaitions également, secondairement, examiner les éventuels effets du genre et du statut. Au niveau du genre, si l'on considère l'allégeance globalement, il est notamment mis en évidence que les hommes sont toujours plus fréquemment partisans de l'utilisation des stratégies passives que ne le sont les femmes, sauf dans le sous-groupe des salariés rebelles, où alors les femmes emploient plus que les hommes le futur favorisant la victime. En considérant maintenant la seule allégeance idéologique, il apparaît que les hommes sont les seuls à mettre en avant la responsabilité morale ou comportementale de la victime, fait qui peut provenir de ce que les femmes sont plus fréquemment victimes d'injustices que les hommes, notamment davantage victimes de harcèlement ( $c f$. Hirigoyen, 2001). Mais nous constatons également que les hommes sont aussi les seuls à parfois proposer l'emploi d'une stratégie active (en l'occurrence l'utilisation de la contestation collective légale). Nous retrouvons donc ici la non contradiction entre le fait de proposer des conduites passives et celui de souhaiter une action permettant de rétablir la justice. Corollairement parlant, cela confirme que «la dénonciation des injustices n'implique pas que les victimes soient perçues comme innocentes et sympathiques» (Dubet, 2006, p382). Quant à l'examen de l'effet genre au niveau de l'allégeance mercenaire, on observe à nouveau que les hommes sont les seuls à employer la responsabilité morale ou comportementale (mais aussi les seuls à envisager une contestation collective légale), et qu'a contrario les femmes sont les seules à suggérer l'utilisation $\mathrm{du}$ futur compensateur, du futur favorisant la victime et de l'impuissance historique. Ainsi, pour les hommes, la victime serait à nouveau responsable de son sort, ce qui signifie que pour les hommes, la formulation de Lerner et Simmons (1966) s'avère exacte: les gens méritent ce qu'ils obtiennent et obtiennent ce qu'ils méritent. A l'inverse, les femmes auraient tendance à évoquer un happy end envers la victime. On peut alors se demander si cette différence ne tient pas aux rôles sociaux respectifs des hommes et des femmes. Plus globaement, si ces résultats mettent effectivement en évidence un effet genre, ils indiquent aussi que cet effet varie selon le type d'allégeance considéré. Enfin, concernant le statut, son effet s'observe surtout en examinant là encore séparément chacun des deux types d'allégeance. Au niveau de l'allégeance idéologique, on constate que les rebelles (qu'ils soient hommes ou femmes) utilisent généralement plus de stratégies passives quand ils sont salariés que lorsqu'ils sont au chômage, l'inverse n'étant généralement observé que chez les allégeants. Et sur le plan de l'allégeance mercenaire, on remarque également que les salariés utilisent plus de stratégies passives que les chômeurs, mais avec une exception qui se concrétise, certes à nouveau chez les allégeants, mais alors uniquement au sein de la population masculine.

\section{Références bibliographiques}

Adams J.S. (1965). Inequity in social exchange. In: L. Berkowitz L. (Ed.). Advances in experimental social psychology. New-York et London: Academic Press, vol.2, 267-299.

Dubet, F. (2006). Injustices, l'expérience des inégalités au travail. Paris: Seuil.

Festinger L. (1957). A theory of cognitive dissonance. Evanston: Row, Peterson.

Gangloff, B. (2005). Réactions différenciées aux injustices professionnelles selon le degré d'adhésion à la norme d'allégeance. Psihologia Resurselor Umane, vol.3 (2), 70-78.

Gangloff, B. (2006). Interiorization of the allegiance norm, and acceptance versus protest facing professional injustices. $26^{\text {ème }}$ Congrès de l'International Association of Applied Psychology (I.A.A.P.). Athènes (Grèce).

Gangloff, B. (2007). Nivel de alineacion de los desempleados como variable determinante de sus reacciones frente a los problemas de desempleo. $31^{\text {ème }}$ Congrès de l'Interamerican Society of Psychology. Mexico (Mexique).

Gangloff, B. (2010). La norme d'allégeance. In: S. Laberon (Ed.). Psychologie et recrutement; modèles, pratiques, normativités. Bruxelles: De Boeck, chap. 4.

Gangloff, B. et Duchon, C. (2007). Quand un idéologue et un mercenaire rencontrent un allégeant et un rebelle: étude de la désirabilité sociale estimée de leurs conduites, $5^{\text {ème }}$ Conférence Internationale de Psychologie Appliquée. Timisoara (Roumanie).

Hirigoyen, M.F. (2001). Malaise dans le travail. Harcèlement moral. Démêler le vrai du faux. Syros, pp.290.

Jones, C et Aronson, E (1973). Attribution of fault to a rape victim as a function of respectability of the 
victim. Journal of personality and social psychology, vol. 26, 3, 415-419.

Kenrick, D.T., Reich, J.W., et Cialdini, R.B. (1976) Justification and compensation: rosier skies for the devalueted victim. Journal of Personality and Social Psychology, 34, 654-657.

Lerner, M.J. (1965). Evaluation of performance as a function of performer's reward and attractiveness. Journal of Personality and Social Psychology, 1, 355-360.

Lerner, M. J. (1980). The belief in a just world: A fundamental delusion. New York, Plenum Press.
Lerner, M.J. et Simmons, C.H. (1966). Observer's reaction to the ,innocent victim": compassion or rejection? Journal of Personality and Social Psychology, 4, 203-210.

Rotter, J.B. (1966). Generalized expectancies for internal versus external control of reinforcement. Psychological Monographs, 80 (609), 1-28.

Steiner, D. D., et Bertolino, M. (2006). The contributions of organizational justice theory to combating discrimination. Cahiers de l'URMIS , $\mathrm{N}^{\circ}$ 10-11, 55-63.

Seligman, M.E.P. (1972). Learned helplessness. Annual Review of Medecine, 23, 407-412. 
Annexe 1: Fiabilité de l'échelle d'allégeance (toutes les probabilités sont inférieures à 0.05).

\begin{tabular}{lcccccc}
\hline \multicolumn{1}{c}{ Dimension } & Nb items & Moyenne & Ecart-type & KR20 & F & P \\
\hline Allégeance-non-allégeance & 12 & 4.79 & 2.048 & 0.78 & 56.648 & $\mathbf{0 . 0 0 0}$ \\
Allégeance (Al+AA) & 6 & 1.47 & 1.867 & 0.82 & 9.178 & $\mathbf{0 . 0 0 0}$ \\
Non-allégeance (RI+RA) & 6 & 3.30 & 1.572 & 0.60 & 52.653 & $\mathbf{0 . 0 0 0}$ \\
Idéologie (Al+RI) & 6 & 2 & 1.739 & 0.70 & 18.145 & $\mathbf{0 . 0 0 0}$ \\
Mercenaire (AA+RA) & 6 & 2.78 & 1.420 & 0.56 & 98.013 & $\mathbf{0 . 0 0 0}$ \\
\hline
\end{tabular}

\section{Annexe 2: les 3 cas}

Cas 1

René est à la recherche d'un emploi depuis plus d'un an. La plupart des CV qu'il envoie restent sans réponse, ou alors il lit toujours la même chose: „Après examen de votre candidature, nous avons le regret de ne pouvoir y donner une suite favorable. Nous sommes cependant persuadés que vous trouverez rapidement un emploi correspondant à vos compétences...". René va bientôt cesser de percevoir les ASSEDIC. Il a suivi plusieurs stages de Techniques de Recherche d'Emploi, il téléphone ou se présente à toutes les adresses qu'on lui indique, mais c'est toujours la même chose: il a trop peu d'expérience ou il en a trop, il a trop peu de formation ou il en a trop. Il ne sait plus quoi faire, il est de plus en plus découragé.

Ce qui a mis le feu aux poudres, ce sont peut-être les derniers entretiens qu'il a eu, pour un poste qui l'intéressait beaucoup. Il a d'abord rencontré un recruteur, puis le chef du service où il y avait le poste, puis encore quelqu'un d'autre... A chaque fois, il a eu l'impression que tout s'était bien passé; d'ailleurs, si ça n'avait pas été le cas, on ne l'aurait pas convoqué à tous ces entretiens. Et puis „vlan”: alors qu'il croit que tout est bon, on lui dit qu'il n'est pas pris. Ou plus exactement quelqu'un lui laisse entendre qu'il est bien le meilleur candidat, mais que le poste est depuis le départ réservé au fils ou au neveu d'untel, et que la procédure était truquée. Ce n'était que „du bidon”. René est abattu. Il n'a plus envie de rien. Il a envie de tout laisser tomber. D'autant qu'il se dit que ce n'est sans doute pas la 1ère fois qu'il se présente pour un poste qui en fait n'est pas disponible. Si c'est toujours comme ça, René pense que ça ne sert à rien de continuer à cherche un emploi.

Cochez les cases en indiquant les réponses que vous attendez d'un bon candidat.

1. Ce refus d'embauche de René vous semble:

$$
\begin{array}{llll}
\text { Totalement juste } & \text { Juste }
\end{array} \quad \text { Injuste } \square \quad \text { Totalement injuste } \square
$$

2. La prochaine offre d'emploi sera la bonne: plutôt d'accord $\square \quad$ plutôt pas d'accord $\square$

3. Puisque René était le plus compétent, ce n'est peut-être pas parce que le poste était „réservé” qu'il n'a pas été pris, mais peut-être aussi à cause de sa personnalité: plutôt d'accord $\square \quad$ plutôt pas d'accord $\square$

4. René n'a peut-être pas adopté les bons comportements pendant les entretiens, sinon, puisqu'il était le plus compétent, il aurait peut-être quand même été embauché:

$$
\text { plutôt d'accord } \square \quad \text { plutôt pas d'accord } \square
$$

5. Puisque c'était une offre d'emploi truquée, René aurait dû porter plainte: plutôt d'accord $\square \quad$ plutôt pas d'accord $\square$

6. René aurait dû faire un scandale et menacer de tout révéler si on ne le prenait pas: plutôt d'accord $\square \quad$ plutôt pas d'accord $\square$ 
7. René ne pouvait rien faire: les recruteurs ont toujours le dernier mot: plutôt d'accord $\square \quad$ plutôt pas d'accord $\square$

8. René aurait dû tout de suite demander à l'Association Régionale des Chômeurs d'intervenir: plutôt d'accord $\square \quad$ plutôt pas d'accord $\square$

9. De toutes façons, dans une entreprise comme celle là, René n'aurait pas été bien. C'est mieux qu'il n'ait pas été pris: il trouvera mieux ailleurs: plutôt d'accord $\square \quad$ plutôt pas d'accord $\square$

Cas 2

Francis a été licencié et recherche un nouvel emploi. Il a un entretien avec son conseiller. Voici son récit:

„J'ai travaillé dans cette agence régionale pendant plusieurs années. J'ai pris volontairement et accepté beaucoup de responsabilités, et j'ai prouvé que j'étais vraiment capable de faire du bon boulot. Là dessus, je n'ai jamais eu aucun reproche, bien au contraire. En fait, les problèmes ont commencé avec l'arrivée d'un nouveau chef d'agence. Je n'accepte pas qu'on me demande n'importe quoi: quand un projet me semble mauvais, ou quand une procédure me semble inefficace, je n'hésite pas à le dire. Si je m'investis dans mon travail, ce n'est pas pour que quelqu'un d'autre, même un chef, prenne des décisions idiotes qui réduisent mes efforts à zéro. J'ai d'ailleurs appris plus tard que mon nouveau chef d'agence n'avait eu son poste que parce qu'il avait beaucoup de relations à la Direction Générale parisienne, et non pas du fait de ses compétences... Mais le problème c'est que j'ai été de plus en plus souvent en conflit avec ce nouveau chef, jusqu'au jour où il m'a convoqué pour un entretien préalable avant licenciement. Il a reconnu que je faisais du bon travail, mais il m'a aussi dit qu'il n'acceptait plus que je conteste ses décisions. J'ai bien essayé de me défendre, mais sans succès: quelques jours plus tard, je recevais ma lettre de licenciement".

Cochez les cases en indiquant les réponses que vous attendez d'un bon candidat.

1. Le licenciement de Francis vous semble:

$$
\text { Totalement juste } \square \quad \text { Juste } \square \quad \text { Injuste } \square \quad \text { Totalement injuste } \square
$$

2. Francis aurait dû porter plainte: plutôt d'accord $\square \quad$ plutôt pas d'accord $\square$

3. Même si Francis est compétent, ce n'est sûrement pas quelqu'un malin: pour ne pas avoir ce type de problème, quand on conteste, il faut le faire avec plus de diplomatie: plutôt d'accord $\square \quad$ plutôt pas d'accord $\square$

4. On peut comprendre que Francis vive très mal d'avoir été licencié. Mais une fois son licenciement décidé, il ne pouvait plus rien faire: les employeurs sont toujours les plus forts: plutôt d'accord $\square \quad$ plutôt pas d'accord $\square$

5. Malgré sa compétence, Francis aurait dû cesser de contester. En continuant ainsi, il ne pouvait qu'avoir des problèmes:

$$
\text { plutôt d'accord } \square \quad \text { plutôt pas d'accord } \square
$$

6. C'est un mal pour un bien, Francis trouvera mieux ailleurs: plutôt d'accord $\square \quad$ plutôt pas d'accord $\square$

7. Lors de l'entretien, Francis aurait dû menacer son chef. Par exemple le menacer de révéler à la Direction Générale toutes les erreurs de gestion commises dans l'agence s'il était licencié: plutôt d'accord $\square \quad$ plutôt pas d'accord $\square$

8. Francis aurait dû faire appel à un syndicat: plutôt d'accord $\square \quad$ plutôt pas d'accord $\square$

9. Ce n'est qu'une mauvaise période à passer. Francis retrouvera certainement bientôt du travail et il oubliera tout ça: plutôt d'accord $\square \quad$ plutôt pas d'accord $\square$ 
Cas 3

Tout se passait bien pour Roger jusqu'au jour où, suite à une opération chirurgicale qui se passe mal, il doit rester plusieurs mois en arrêt maladie. A son retour, il apprend que, pendant son absence, il a été remplacé par quelqu'un de moins compétent que lui, mais qui est le fils de l'un des chefs de service de l'entreprise. Le patron de l'entreprise propose alors à Roger un licenciement à l'amiable, ce que Roger refuse.

Depuis, sa vie professionnelle est un enfer. On ne lui transmet plus aucune information, on l'exclut de toutes les réunions; on lui donne des ordres sans cesse contradictoires, pour des tâches idiotes et sans aucun intérêt. En fait, on ne lui donne plus aucun véritable travail. Il est „mis au placard”. On veut le faire craquer pour qu'il quitte l'entreprise de lui-même...

Cochez les cases en indiquant les réponses que vous attendez d'un bon candidat.

1. Ce qui arrive à Roger vous semble:
Totalement juste $\square$
Juste $\square$
Injuste
Totalement injuste $\square$

2. Roger ne peut rien faire: soit il démissionne et se retrouve au chômage, soit il subit, même si c'est dur. De toutes façons, on ne gagne jamais dans une épreuve de force avec son employeur: plutôt d'accord $\square \quad$ plutôt pas d'accord $\square$

3. Roger est sans doute compétent, mais aussi quelqu'un de „buté”, sinon on lui donnerait à nouveau un travail normal:

plutôt d'accord $\square \quad$ plutôt pas d'accord $\square$

4. Si Roger n'arrive pas à retrouver une situation normale, c'est sans doute que malgré sa compétence il n'adopte pas les bons comportements: plutôt d'accord $\square \quad$ plutôt pas d'accord $\square$

5. Roger devrait avertir l'inspection du travail: plutôt d'accord $\square \quad$ plutôt pas d'accord $\square$

6. Roger devrait lui aussi rendre la vie impossible à ses chefs, jusqu'à ce qu'ils acceptent de lui donner à nouveau du travail: plutôt d'accord $\square \quad$ plutôt pas d'accord $\square$

7. Ce licenciement aura un côté positif et montrera à Roger la vraie mentalité de son employeur. Mieux vaut que Roger parte au plus vite: il trouvera certainement mieux ailleurs: plutôt d'accord $\square \quad$ plutôt pas d'accord $\square$

8. Roger devrait contacter un syndicat: plutôt d'accord $\square \quad$ plutôt pas d'accord $\square$

9. Roger doit seulement attendre un peu: dans quelque temps, tout finira par rentrer dans l'ordre: plutôt d'accord $\square \quad$ plutôt pas d'accord $\square$ 